\title{
Development of IQRF Technology: Analysis, Simulations and Experimental Measurements
}

\author{
Radek Fujdiak $^{1,2}$, Petr Mlynek ${ }^{1,2}$, Lukas Malina ${ }^{1}$, Milos Orgon ${ }^{3}$, Jan Slacik ${ }^{1}$, Petr Blazek ${ }^{1}$, Jiri Misurec ${ }^{1}$ \\ ${ }^{1}$ Department of Telecommunication, Brno University of Technology, \\ Technicka St. 12, 61600 Brno, Czech Republic \\ ${ }^{2}$ Peoples' Friendship University of Russia, \\ Miklukho-Maklaya St. 6, 117198 Moscow, Russian Federation \\ ${ }^{3}$ Department of Telecommunications, Slovak University of Technology in Bratislava, \\ Ilkovicova St. 3, 81219 Bratislava, Slovak Republic \\ fujdiak@vutbr.cz
}

\begin{abstract}
Recent years brought many changes, which accelerated the development of different communication approaches and technologies. Nowadays, wireless technologies become the accelerators for a wide range of new applications. This paper focuses on the latest evolution of the promising wireless communication technology IQRF. For this aim, an indepth analysis together with experimental measurements and simulations are provided. We provide original results in selected indoor and outdoor scenarios, in which the important communication parameters and technological limitations are highlighted. Last but not least, the application field of IQRF is established together with a comparison with other relevant technologies.
\end{abstract}

Index Terms-Mesh networks; Wireless communication; Wireless sensor networks; Telemetry.

\section{INTRODUCTION}

The origins of wireless communication can be found in fire beacons, smoke signals, and others [1]. In the 19th century, the wireless photo-phone [2] and the wireless telegraph were invented [3]. Nowadays, the communication technologies change rapidly. The recent years brought new applications as Internet of Things (IoT) [4]; Machine-toMachine communication (M2M) [5]; smart and intelligent use-cases, i.e., Smart Grid [6], Smart City [7], Smart Home [8]; together with the new industrial revolution Industry 4.0 [9]; and many others. For most of them, the wireless communication became the accelerator [10]. This also caused the evolution of the wireless technologies, which results in many different approaches. There are many variations, i.e., in the frequency band (sub-GHz, over-GHz), band licensing (licensed or unlicensed band), network topology (star topology, mesh topology, tree topology), frequency modulation, channel bandwidth, security, powerusage, and others [1]. The low-power mesh, among others, is one of the promising approaches, used for a wide range of

Manuscript received 16 April, 2018; accepted 30 August, 2018.

The National Sustainability Program under grant LO1401 and the Czech Ministry of the Interior under grant no. VI20172019057 financed the research described in this article. For the research, the infrastructure of the SIX Center was used. The publication was prepared with the support of the RUDN University program 5-100. applications, i.e., ad-hoc sensor networks, due to its robustness, resilience and range extension [11]-[13]. In this paper, we focus on the IQRF technology (Fig. 1). This technology was established by Microrisc s.r.o. company. In late 2017, the company separated and the subsidiary company IQRF Tech was created, which took over the IQRF brand.

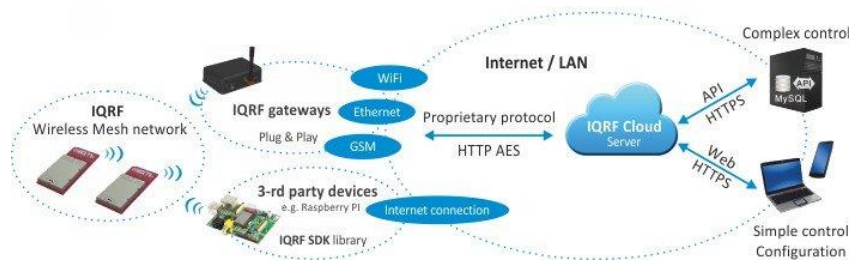

Fig. 1. The IQRF technology ecosystem with application, access and transport part (Source [14]).

We believe that this technology might be a promising solution for many different applications. However, the technology changed markedly and very fast over the last years. This is, among the others, the reason for the missing independent scientific results, which would help to determine the parameters, advantages, and the limits of this technology. To provide a more detailed overview of this technology, we provide an in-depth analysis, where the historical development together with the current version of the technology is discussed. Moreover, we have reviewed the published scientific sources and discussed their findings Further, we provide also original results from our simulation and measurements in the selected indoor and two outdoor scenarios (urban-area and open-area). Further, the important communication parameters, approach advantages, and technological limitations are highlighted. Last but not least, the application field of IQRF is established, together with a comparison with other relevant technologies.

The rest of the paper is organized as follows: Section II provides an in-depth analysis of the IQRF technology evolution. Moreover, we also give a clear overview of recent scientific works in this area. Section III introduces pre-settings for our simulations and experimental measurements in indoor and outdoor scenarios. Further, the main findings and results are included in Section IV. The 
discussion and comparison with other related technologies are provided in Section IV. Finally, Section V summarizes our conclusions.

\section{ANALYSIS OF IQRF TECHNOLOGY DEVELOPMENT}

Since 1991, IQRF technology is an established part of the ICT world. However, it has changed remarkably over the last years to be able to compete on the market. In its beginnings, the IQRF was meant to be a simple wireless home automation technology [15]-[17]. However, the technology evolved together with the market and the increasing needs of the customers (users). Since 2004, there have been experiments and development for the mesh topology [18]. The complete mesh topology for IQRF was introduced in early 2008 (OS version 2) [19]. The first paper about the IQRF mesh topology was published already in early 2009 and discussed the advantages of wireless communication platform [20]. The provided mesh topology had a significant impact on the future development of the technology. It opened up new directions in the market as well as in the scientific research. During the last years, many different use-cases for the IQRF were introduced, i.e., home automation [21]-[23]; radio telemetry at mining dumps [24]; wireless sensor networks with internet protocol interoperability [25]; distributed temperature and humidity measurement [26], [27]; underground large-scale remote monitoring [28]; detection of construction inclination [29], racehorse monitoring [30]; erosion of rock formation measurements [31]; city use-case - public light management [32] or intelligent parking [33]; laboratory and student training in sensor networks [34], [35]; and many others.

Nowadays, the intelligent automation, sensor networks, telemetry systems, remote measurements, environmental monitoring or other mentioned applications are already widely spread tools to obtain the information used in advanced analytical algorithms, decision making systems or control systems. This has also been the reason due to which IQRF significantly evolved during the last years and improved many of its crucial parameters. Table I displays the important milestones achieved during the past years of IQRF from its early time to the current shape.

TABLE I. THE MILESTONES OF THE IQRF DEVELOPMENT.

\begin{tabular}{|c|c|c|}
\hline Year & Description & Source \\
\hline 1991 & The IQRF Technology was founded. & {$[15]-[17]$} \\
\hline 2008 & The first MESH IQRF topology was introduced. & {$[19]$} \\
\hline 2011 & $\begin{array}{c}\text { The IQMESH was introduced, supporting 65 } \\
\text { thousands of devices, 240 hops. New power } \\
\text { management with 35 } \mu \text { A. }\end{array}$ & {$[36]$} \\
\hline 2013 & $\begin{array}{c}\text { The DPA (Direct Peripheral Access) was } \\
\text { introduced in the version of v1.00. }\end{array}$ & {$[37]$} \\
\hline 2014 & $\begin{array}{c}\text { New DPA version developed and implemented } \\
\text { (v2.00), FRC method (Fast response command) } \\
\text { for group messaging was introduced. }\end{array}$ & {$[38]$} \\
\hline 2015 & $\begin{array}{c}\text { Bit-rate 19.8 kb/s and minor improvement for } \\
\text { FRC and DPA. }\end{array}$ & {$[39]$} \\
\hline 2016 & $\begin{array}{c}\text { IQRF starts to support the unlicensed band- } \\
\text { frequencies 433 MHz and 916 MHz. }\end{array}$ & {$[40]$} \\
\hline & $\begin{array}{c}\text { The new version of OS introduced (4.00D), } \\
\text { packet structure changed, added encryption via } \\
\text { AES-128, a new version of DPA (v3.00), deep- } \\
\text { sleep mode implemented. }\end{array}$ & {$[41]$} \\
\hline
\end{tabular}

The IQRF currently has the following main parameters
[14]: (i) unlicensed wireless radio technology (supporting frequencies $868 \mathrm{MHz}, 916 \mathrm{MHz}$ and $433 \mathrm{MHz}$ ); (ii) P2P or MESH topology; (iii) ultra-low-power (sleep mode hundreds of $\mathrm{nA}$; transmission - based on mode, average hundreds of $\mu \mathrm{A}$ ); (iv) low throughput $(19.836 \mathrm{~kb} / \mathrm{s}$ or $19.2 \mathrm{~kb} / \mathrm{s}$ ); (v) byte oriented protocol DPA for services and peripherals control; (vi) low to medium range (considering small antennas - tens of meters in buildings, hundreds of meters in open space); (vii) security (nodes are bound to the network via password, network encryption via AES-128 with self-distribution of the keys, optional user encryption via AES-128, message checksum CRC-16, and block checksums). Moreover, the IQMESH supports different MESH routing algorithms (full-mesh routing, reduced mesh routing, optimized mesh routing and others) and up to 240 devices in a single network with a maximum of 240 hops per packet (33 hops in 1 seconds with DPA method) [14].

As previously said, many new technologies and approaches were recently introduced, including new trends of the smart grid, smart city, smart home, smart factory and others. The Low-Power Wide Area Networks (known as LPWAN) are among the most discussed. The principles of LPWAN are known already since the late 1990s, where the AlarmNet Network was introduced [42]. The current trend of LPWAN started with SigFox in 2009 and continued with many new technologies as LoRaWAN, Ingenu, Waviot, NB-IoT, and others. However, many of these technologies are still in the phase of their early development. The first relevant scientific papers have been published in late 2015 . The paper [43] shows some similarities and differences between main LPWAN technologies (LoRaWAN and SigFox) and the IQRF. The article contains a short introduction with a simple comparison of selected parameters based on the technical documentation for LPWAN and IQRF. However, the main conclusion of the paper is based on possible interferences caused by channel occupation. The authors have used a mathematical (theoretical) approach and computed the possible occupation of the channels based on the technical documentation. Another interesting part of the article is also the comparison of the IQRF with LPWANs. However, we believe that the IQRF rather belongs to the type of technology which is the so-called "Low Power Local Area Network" (LPLAN) or "Low Power Metropolitan Area Network" (LPMAN) than to the LPWAN type. This thought is supported by the technical parameters of IQRF; the lowpower attribute with the short/medium communication range (LPLAN - indoor range amounts to tens of meters; LPMAN - outdoor range amounts to hundreds of meters) [41]. Moreover, IQRF is not offering operator-based connectivity to single sensors or groups of sensors over large-scale geographical areas; instead, it attempts to create more localbased sensor nests with a higher density of sensors. The LPWANs are covering a certain part of the use-cases of IQRF, but the most appropriate technology for a comparison is the private LoRaWAN from this group (or proprietary LoRa-based systems). Moreover, there are technologies with a similar approach, i.e., ZigBee, BlueTooth [44], or even Wi-Fi (Wi-Fi HaLow) [45]. However, the comparison with these technologies is missing in the article, same as a 
clear insight into the IQRF field. In the following, we provide simulations supported by experimental measurements of selected scenarios, which should provide a clear idea of the parameters and limitations of the IQRF. Additionally, we provide a comparison with related technologies, which should help to establish the differences between the IQRF and the other approaches towards the same field.

\section{Pre-Sets For Simulations AND MEASUREMENTS IN DIFFERENT SCENARIOS}

We are considering three main scenarios for the simulations and measurements: (i) indoor; (ii) outdoor signal (open space); and (iii) outdoor (urban area). The simulation model for the first scenario (Fig. 2) is based on a real flat, which is used for real experimental measurements.

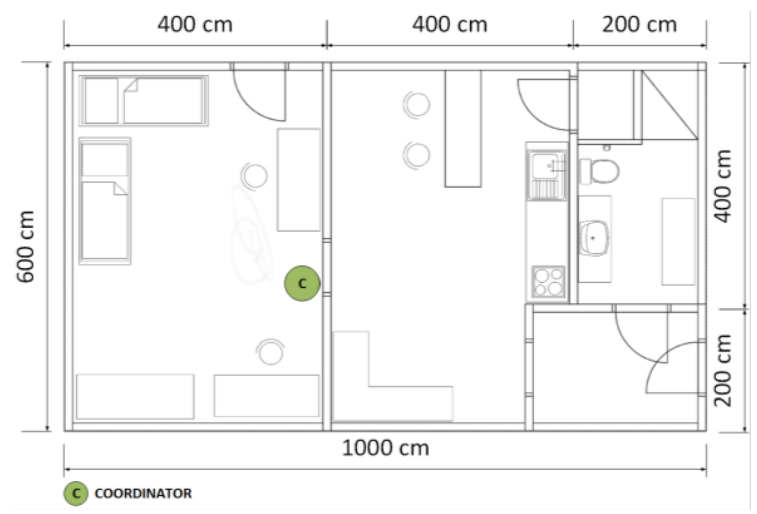

Fig. 2. The considered indoor/outdoor environment simulations and experimental measurements of IQRF signal propagation.

For the indoor simulations, the Remcom Wireless InSite software has been used (professional analytical, simulation and modelling software for radio signal propagation) [46]. The empirical model is based on the frequency, antenna, and material/free-space propagation - wall count. The simulation pre-settings were as follows: $18 \mathrm{~cm}$ thick walls (material: concrete, permittivity $\varepsilon_{\mathrm{r}}=7.0$ ), the doors made from wood $\left(\varepsilon_{\mathrm{r}}=5.0\right)$ and the windows made from glass $\left(\varepsilon_{\mathrm{r}}=\right.$ 2.4). The frequency of $868 \mathrm{MHz}$ with the sinusoid signal (1 $\mathrm{MHz}$ bandwidth) is used, linear dipole antenna with vertical polarization (Voltage Standing Wave Radio, VSWR $=1.00$ with maximum gain $1.740 \mathrm{dBi}$ - used for TX/RX, and transceiver with $11 \mathrm{dBm}$ ). The room is divided into the classical grid of 720 points with a spacing of $0.25 \mathrm{~m}$.

For the outdoor simulation, the Link Technology Tower Coverage software was used (professional analytical, simulation and modelling software for outdoor signal propagation) [47]. This software works with a real map background and heights of the buildings. The pre-settings for the simulation were based on the devices used for the experimental measurements - TR-72D and GW-ETH-02A. The parameters are as follows: the generic omnidirectional antenna (height of 30 meters for TX and 5 meters for RX); the P2P connection was used; the antenna gain was 1.740 $\mathrm{dBi}$ and power $11 \mathrm{dBm}(\mathrm{TX} / \mathrm{RX})$; sensitivity $-104 \mathrm{dBm}$. The areas for the outdoor scenarios are displayed in Fig. 3.

For the experimental measurements, the following equipment was used: (i) transceivers - TR-72D (868 MHz, sensitivity $-104 \mathrm{dBm}, \mathrm{PCB}-$ printed circuit board antenna)
[48]; (ii) gateway - GW-ETH-02A (100 MB Ethernet gateway, PCB antenna, other parameters as TR-72D) [49]; and (iii) cloud - IQRF CIS Cloud [49]. The first indoor scenario was measured as $\mathrm{P} 2 \mathrm{P}$ in the $0.25 \mathrm{~m}$ steps grid (similar to our introduced indoor simulation). The outdoor scenarios were measured in significant distance steps.

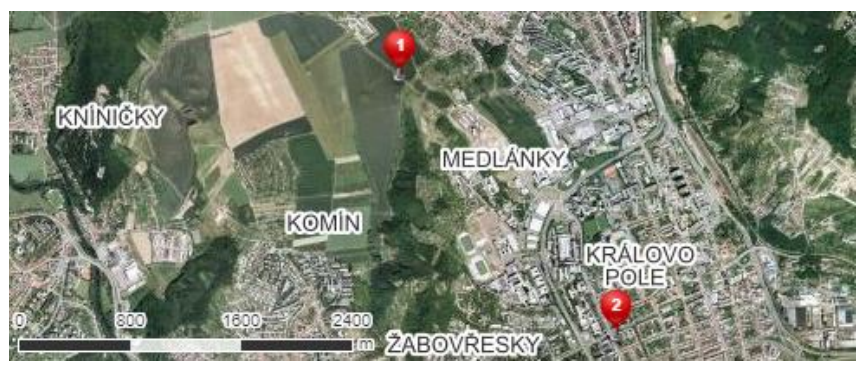

Fig. 3. The selected location in Brno, the Czech Republic for outdoor simulations/measurements: (1) open-space area; (2) urban area.

Besides the three main scenarios, several side demonstration measurements have been done: (i) latency of end-to-end E2E connection (device-gateway-cloud); (ii) long-term temperature measurements; (iii) sensitivity for communication congestion; (iv) tests for network convergence. The topology in the case of (i) and (ii) is a simple two-point indoor topology with free-space communication with a distance of four meters. Moreover, we also measure the latency of mesh topology (Fig. 4) in scenario (i). The topology for scenario (iii) is displayed in Fig. 4 (full-mesh topology) and the topology for scenario (iv) is shown in Fig. 5.

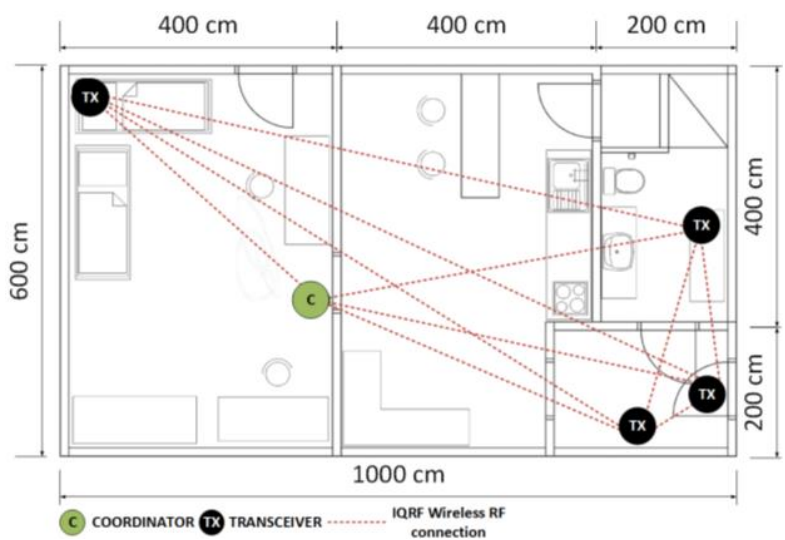

Fig. 4. The considered topology for measuring the impact of network congestion on the used devices and gateway and latency in MESH topology.

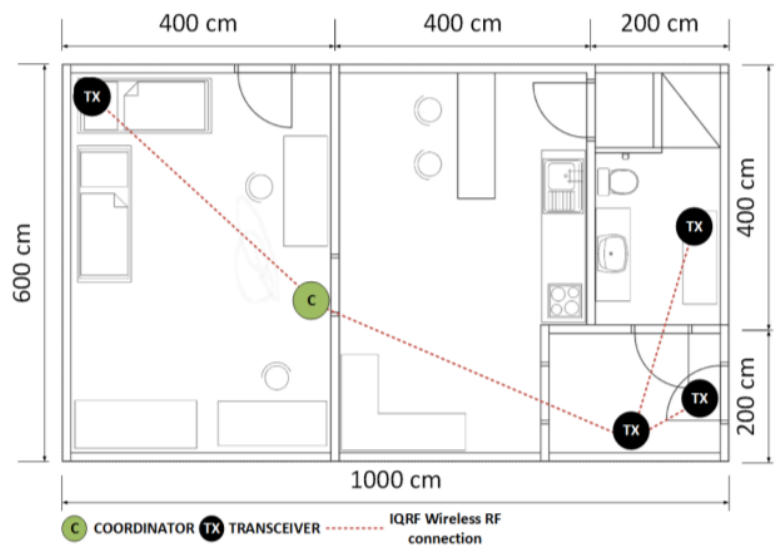

Fig. 5. The considered topology for measuring the functionality of convergence algorithm in IQRF technology. 
The topology for side measurements (iv) was picked to simulate a more critical situation (Fig. 5). The possible outage of the right-wing transceiver might result in an outage of $75 \%$ of the devices in the network without functional convergence system.

\section{Simulation And Measurements Results}

\section{A. The Indoor Scenario}

The main results from the simulation are shown in Fig. 6. The propagation signal strength (RSSI) ranged from $-107 \mathrm{dBm}$ to $-4 \mathrm{dBm}$ and the sensitivity was $-104 \mathrm{dBm}$. The room with the coordinator is just free-space propagation with RSSI from 0 to $-50 \mathrm{dBm}$. Further, the first wall shows a significant decrease of RSSI to value from $-25 \mathrm{dBm}$ to $-75 \mathrm{dBm}$. The entrance hall was covered with RSSI from -50 to $-100 \mathrm{dBm}$. Finally, the small closet and bathroom were already on the border of sensitivity (RSSI value from $-75 \mathrm{dBm}$ to $-100 \mathrm{dBm})$.

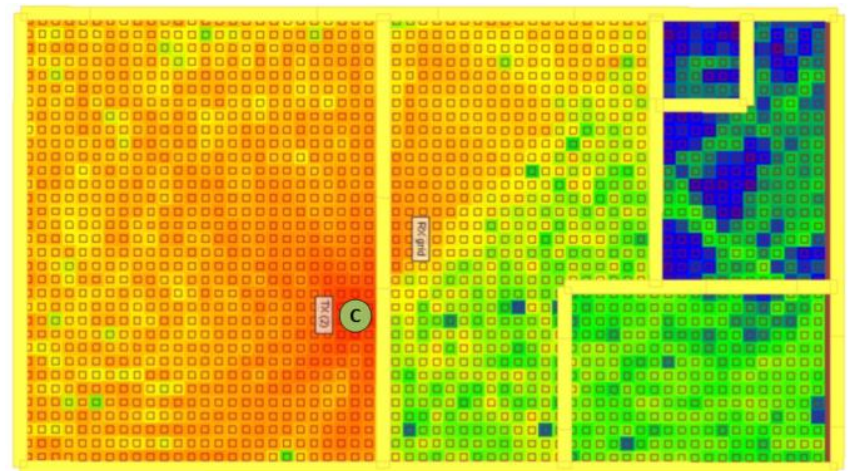

\section{$-107 \mathrm{dBn}$}

(C) COORDINATOR

Fig. 6. Simulation for signal propagation in the indoor scenario.

The results were not in favour of IQRF and also not accurate (compared with experimental measurements). The experimental measurements show different better results (Fig. 7). The first room was covered by the signal of -40 to $-60 \mathrm{dBm}$ RSSI. The RSSI in the second room stayed nearly unchanged (from $-40 \mathrm{dBm}$ to $-60 \mathrm{dBm}$ ). Further, the small closet and bathroom were covered with RSSI from $-60 \mathrm{dBm}$ to $-70 \mathrm{dBm}$.

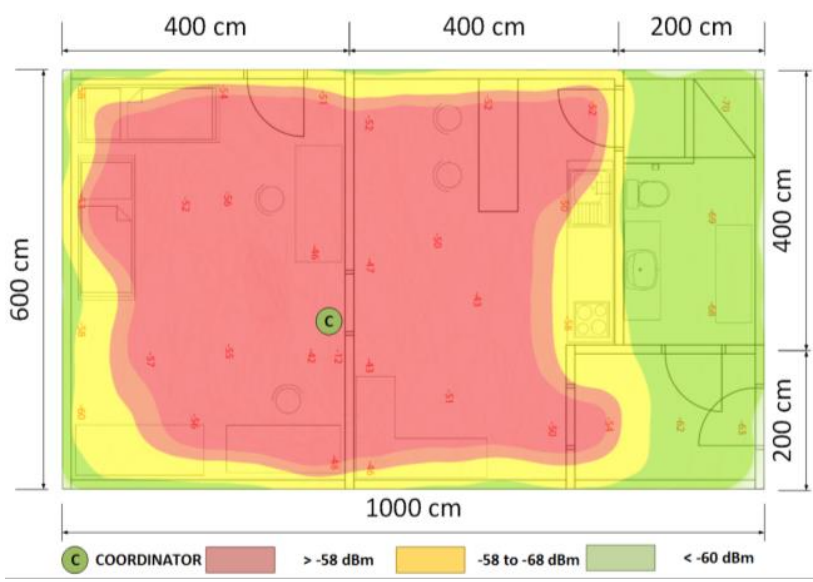

Fig. 7. Real measurement of signal propagation in the indoor scenario.

In the simulation, each wall caused an average RSSI loss of $25 \mathrm{~dB}$ (average $20 \mathrm{~cm}$ concrete wall attenuation at
$900 \mathrm{MHz}$ has in general $23 \mathrm{~dB}$ [50]). However, the real measurements of IQRF show that each wall reduces the RSSI only by $10 \mathrm{~dB}-15 \mathrm{~dB}$ (the difference is caused by general $868 \mathrm{MHz}$ model, where IQRF has advance modulation/correction codes, and others). This shows very good indoor signal propagation, which is a significant advantage compared to the over- $\mathrm{GHz}$ technologies, i.e., $2.4 \mathrm{GHz}$ frequency band as $\mathrm{Wi}-\mathrm{Fi}$, where the wall attenuation is significantly higher [51]).

\section{B. The Outdoor Open-Space Scenario}

The first outdoor simulation was done in the open-area scenario. The main results are depicted in Fig. 8. The maximum communication distance over the open-area was over $1 \mathrm{~km}$ in the west side (left). The east side (right) had significant attenuation caused by the beginning of the urban area and the hilly terrain. The terrain and building obstacles significantly reduce the communication range to $200 \mathrm{~m}-$ $400 \mathrm{~m}$.

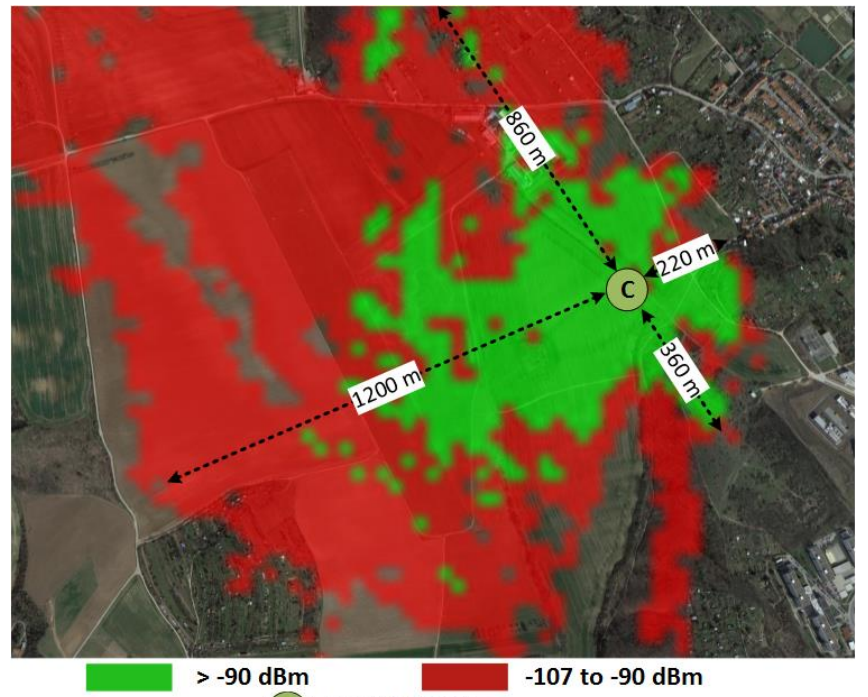

(C) COORDINATOR

Fig. 8. Simulation of signal propagation in the outdoor open-space scenario (Medlanecky kopec, Brno area, Czech Republic).

The real measurement (Fig. 9) shows that the simulation was sufficiently accurate.

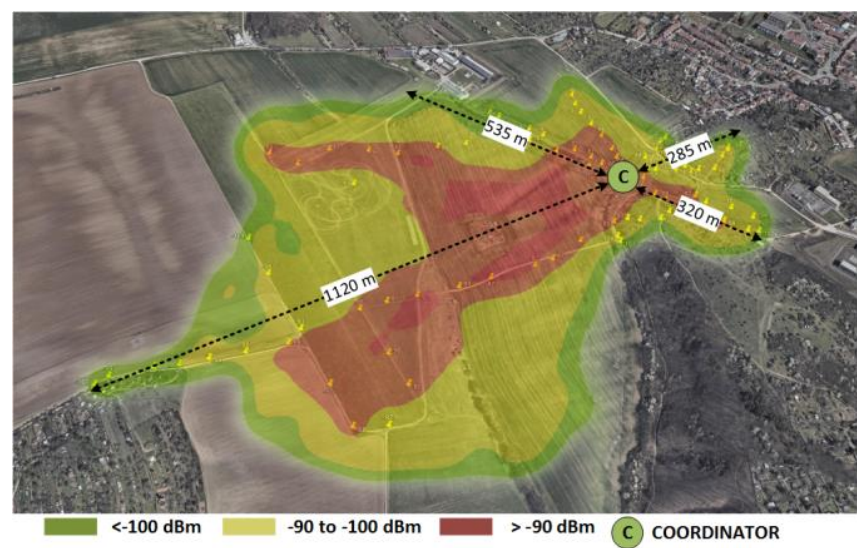

Fig. 9. Real measurement of signal propagation in the outdoor open-space scenario (Medlanecky kopec, Brno area, Czech Republic).

The results show slightly worse results than predicted by the simulation. The real maximum communication range was $1120 \mathrm{~m}$, again in the open area (left side). However, the 
north (up) range was $535 \mathrm{~m}$, where the attenuation was caused by nearby farm-houses. However, the results for the east (right) and south (down) section were approximately $300 \mathrm{~m}$.

The simulation and the measurement show the limitation of the IQRF technology. Each terrain obstacle immediately and significantly reduces the communication range. However, the results accorded to the technicaldocumentation, wherein open-area should IQRF should provide hundreds of meters of communication range. Moreover, the line-of-sight range is over $1 \mathrm{~km}$, which might be advantageous in the case of covering the MAN areas.

\section{The Outdoor Urban Area Scenario}

The third main scenario was in the outdoor urban area. The simulation shows as expected worse results compared to the open-area scenario (Fig. 10). The communication range was reduced approximately to $100 \mathrm{~m}$. The maximum communication distance was $165 \mathrm{~m}$.

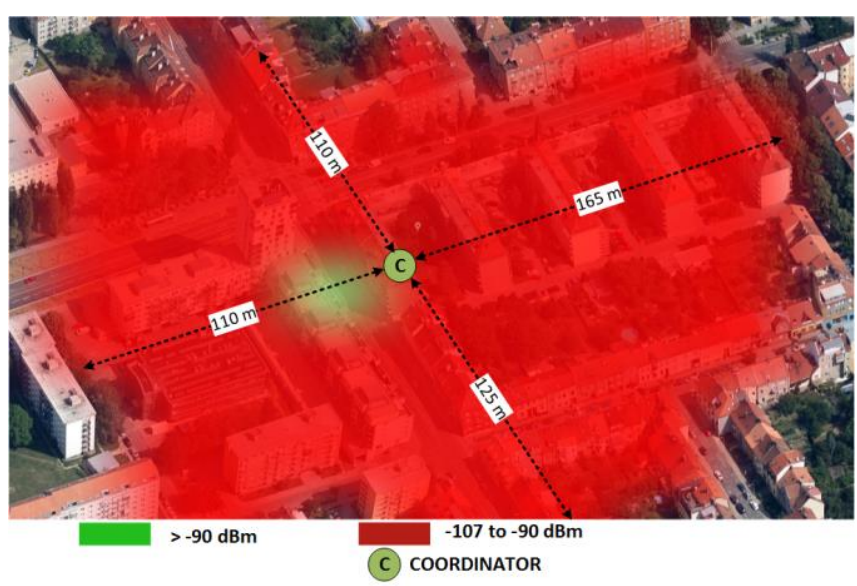

Fig. 10. Simulation of signal propagation in the outdoor urban area scenario (Purkynova St. 35e, Brno, Czech Republic).

The real measurements partially confirmed the simulation results (Fig. 11). Again, the real measurement results were inferior to the simulation.

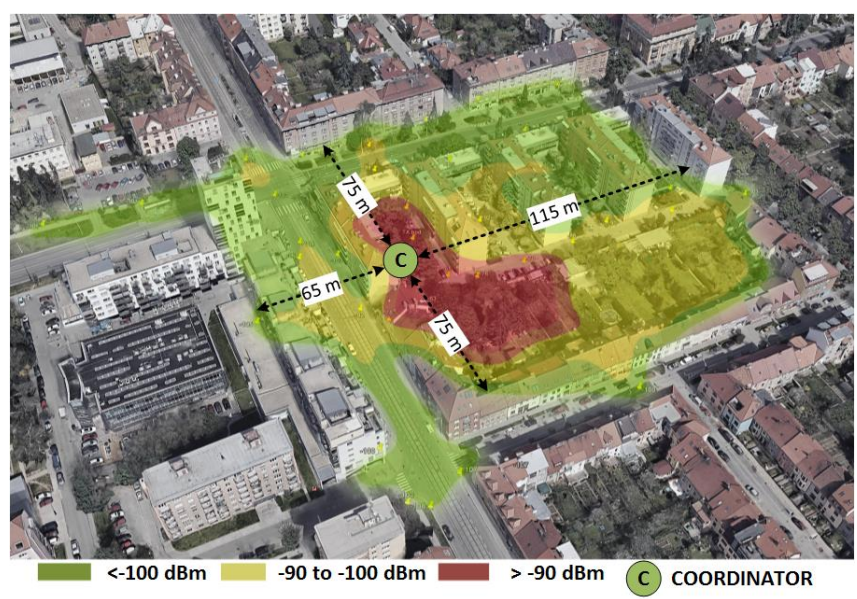

Fig. 11. Real measurement of signal propagation in the outdoor urban area scenario (Purkynova St. 35e, Brno, Czech Republic).

However, the communication range was approximately $80 \mathrm{~m}$. The maximum communication distance was $115 \mathrm{~m}$. However, the real measurements show slightly better results in the case of attenuation. The simulation results show in most of the covered area only border RSSI, but the real measurements show in average $10 \mathrm{~dB}$ better results (i.e., the area over $-90 \mathrm{dBm}$ is more extensive).

The third scenario revealed the real limitations of the IQRF technology. The buildings attenuation in highly urbanized areas has a significant impact on the signal propagation of IQRF technology. The communication range is $90 \%$ reduced, compared with the open-area results. Real communication range in the urban area will be in most cases tens of meters. The signal propagation should be enough for LAN use-cases with significant limitations in MAN usecases. The IRQF technology is not appropriate for WAN use-cases, however, it is not meant to be.

\section{Results of Side Measurements}

\section{1) E2E latency measurement}

We measured the E2E latency (device-gateway-cloud) to investigate its suitability for applications, in which the delay might be a crucial parameter, i.e., crisis management, smart grid control applications, and others. The experiment duration was more than 20 hours. The latency had decreasing linear character (Fig. 12). The average jitter was two seconds and the average latency $16 \mathrm{~s}$. The messageperiod was 1 minute. 1226 messages were sent and received, which means $100 \%$ success rate.

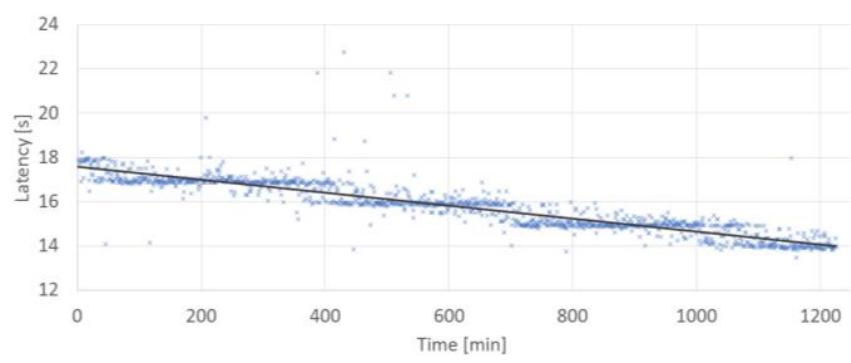

Fig. 12. Real measurement of E2E latency (device-gateway-cloud).

The gateway was connected via a standard internet provider without strict SLA (Service-Level-Agreement). We also use only a free-to-use cloud service without priority processing. For this reason, we also measure the latency as P2P (device-gateway), see Fig. 13. The experiment duration was over 80 minutes.

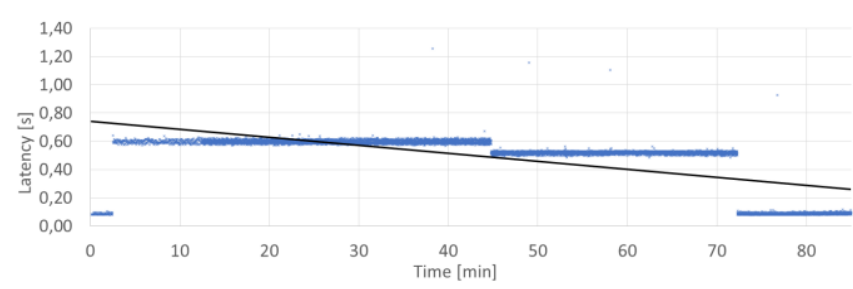

Fig. 13. Real measurement of P2P latency (device-gateway).

The latency had again decreasing linear character. However, the measurement shows that the latency between gateway and device was $400 \mathrm{~ms}$ in average and under one second. As we can see, the gateway and service providers caused the higher delay. To investigate the mesh-topology and reveal the hidden delay, we also measured in the topology displayed in Fig. 5. Latency is computed as average from all four nodes. The delay was measured for the gateway to cloud requests (GW-Cloud) and transceiver to gateway requests (TX-GW). The message periodicity was one per minute. Figure 14 shows the results of our 
experimental measurements. The graph shows initial higher latency (up to first 20 minutes), two peaks (at approx. 10 minute and 70 minute) and stable $20 \mathrm{~s}$ delay (the period from 20 minute to 70 minute). The initial delay was caused by the transport network (gateway-cloud side) same as the two peaks. However, the average latency was $25 \mathrm{~s}$, which approximately corresponds to the simple P2P measurement (Fig. 12).

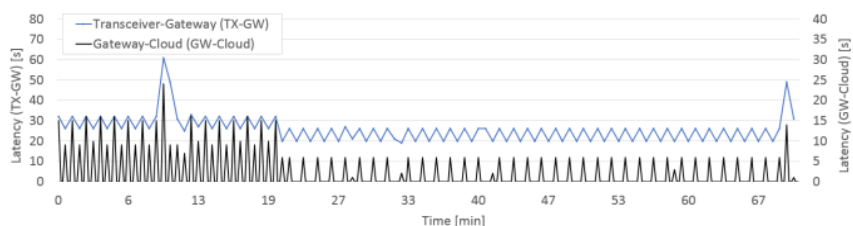

Fig. 14. Real measurement of P2P latency (device-gateway).

\section{2) Impact on the network congestion}

The purpose of this scenario was to evaluate via stress test the parameters of IQRF technology in full MESH topology (Fig. 4). The results of this test are displayed in Fig. 15. The message periodicity was one per 30 seconds for each device. The experiment duration was longer than 90 minutes. The message requests were made until the 80 minute of the measurement (after the 80 minute the message requesting was stopped). The graph (Fig. 15) for transceiver-gateway latency has linear growth characteristic until 85 minutes. The latency reaches 120 seconds after the first 10 minutes of the experiment. After 30 minutes, the latency starts to be crucial and takes more than 300 seconds. However, the maximum reached latency was more than 1100 seconds. The message requests were stopped at the 80 minute, but we can still see that the graph constantly grows. Thereafter, the latency starts to decrease. Moreover, the latency on the gateway-cloud side was fluctuating between 0 seconds to 50 seconds (the average was 15 seconds). Compared with the side-scenario (ii), we can see critical latency growth for transceiver-gateway (growth nearly 95 $\%$ ) and significant for gateway-cloud (growth more than 50 $\%)$.

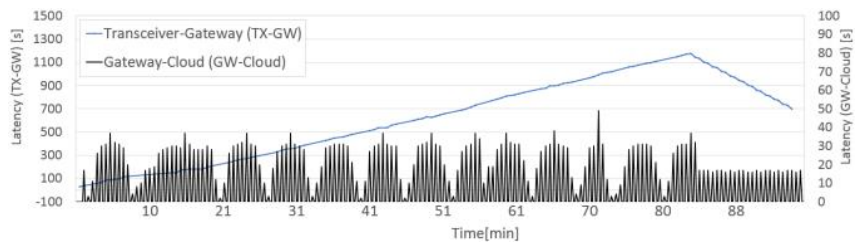

Fig. 15. Real measurement of impact on network congestion.

\section{3) Convergence test measurement}

The topology of the convergence test was as in Fig. 5. As was said, the disconnection of the right main node would cause an outage of $75 \%$ of network transceivers. First, the functionality of the network from Fig. 5 was tested. The signal strength on the last right two devices was $-70 \mathrm{dBm}$. After disconnecting the main right node, the network converges under one second into topology displayed in Fig. 16. Surprisingly, the nodes connect to the most left node and not to the coordinator. However, the communication was established and without loss-rate. Further, the signal strength on both converged devices decreased to $-90 \mathrm{dBm}$. This test proves a resilience of IQRF against sudden outage.

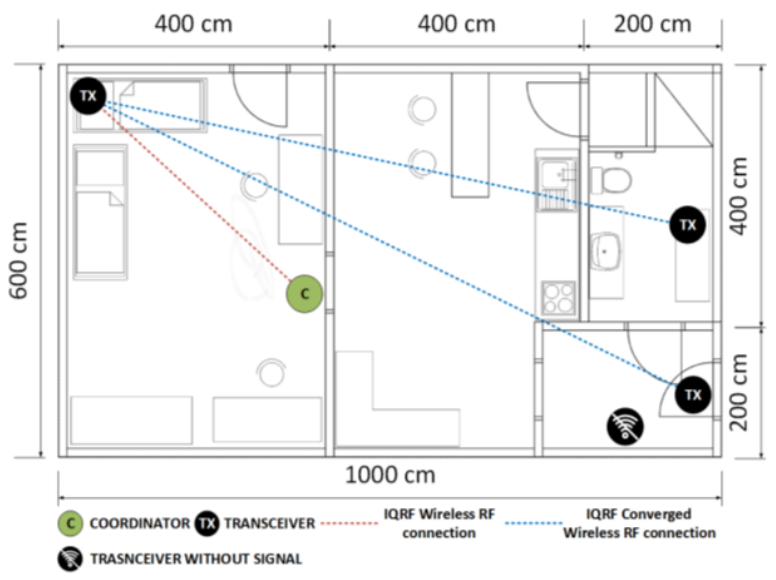

Fig. 16. The converged network after the disconnection of the main node

\section{4) Long-term temperature measurements}

In the last scenario, we validate the IQRF technology for long-term telemetry use-cases (simple P2P connection). We measure on-sun temperature over two days. The measurement was sufficiently accurate, when the $50{ }^{\circ} \mathrm{C}$ peak was caused by straight solar radiation (Fig. 17).

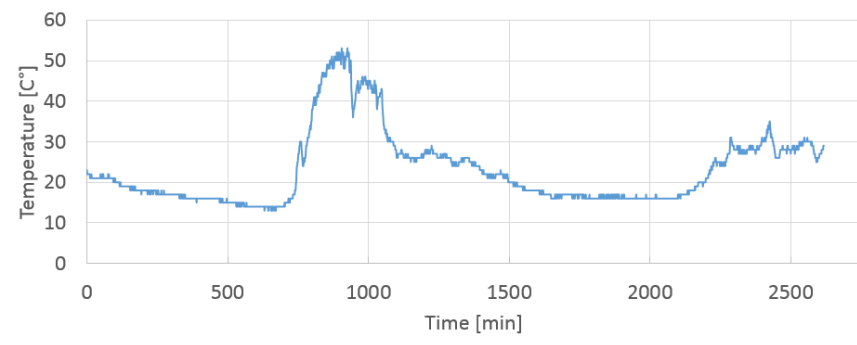

Fig. 17. Long-term P2P measurements of on-sun temperature.

The success rate for the experiment period was $100 \%$.

\section{DISCUSSION}

The results of our measurements and simulations show the main parameters of IQRF technology. The main advantages of this technology lie in:

- good indoor and free-space signal spread, main experiment (i) and (ii),

- good resistance against output and functional convergence methods, side experiment (iii),

- stable parameters for long-term measurements, side experiment (iv).

These parameters move the IQRF technology to the usecases: intelligent public light management, smart parking, home automation systems, and others non-critical ad-hoc or periodical sensor/measurement applications. However, there are also limitations:

- a limited number of nodes in the network (240),

- the strong impact of attenuation in an urban area, main experiment (iii),

- high impact of communication congestion on the network latency - side experiment (ii), and higher general latency up to hundreds of ms - side experiment (i),

- average security without sufficient and clear keymanagement (insufficiently described).

Together this information excludes the IQRF technology 
from the high data rate IoT applications, real-time sensor/telemetry networks, and crucial applications, i.e., crisis management systems. Moreover, the comparison with other technologies is displayed in Table II (BLE for Bluetooth). IQRF offers longer range, higher sensitivity and better power-management (in exchange for lower throughput and higher latency). However, the ZigBee and BLE are more PAN/LAN technologies than LAN/MAN.

TABLE II. COMPARISON OF IQRF MESH SOLUTION WITH RELEVANT SELECTED TECHNOLOGIES (EUROPE).

\begin{tabular}{|c|c|c|c|}
\hline & IQRF & ZigBee & LP BLE \\
\hline Frequency [MHz] & $433 / 868$ & $868 / 2400$ & 2400 \\
\hline Throughput [kbs] & $<20$ & up to 250 & up to $2 \mathrm{Mbit} / \mathrm{s}$ \\
\hline Sensitivity [dBm] & -104 & -102 & -93 \\
\hline Range [m] & up to 1000 & 291 & 100 \\
\hline Latency [ms] & 400 & 20 & 6 \\
\hline Consumption & up to $15 \mu \mathrm{A}$ & $<30 \mathrm{~mA}$ & $<15 \mathrm{~mA}$ \\
\hline Security & AES-128 & $128 \mathrm{~b}$ key & AES-128 \\
\hline Topology* & $\mathrm{s}, \mathrm{t}, \mathrm{m}$ & $\mathrm{s}, \mathrm{t}, \mathrm{m}$ & $\mathrm{m}$ \\
\hline Sources & \multicolumn{3}{|c|}{$[14],[44],[52]$} \\
\hline
\end{tabular}

Based on this comparison, we identified another two relevant technologies. As previously said in Section II, the technologies Wi-Fi HaLow and private LoRaWAN might be the competitors. The comparison with Wi-Fi MESH (IEEE 802.11s) and Wi-Fi Halow (IEEE 802.11ah) is displayed in Table III. However, the Wi-Fi MESH is not low-power and it is suitable for higher-throughput LAN scenarios. The Ha-Low is LAN/MAN technology with lower energy management, but it still does not allow a full run on battery for longer period. The IQRF shows also here its uniqueness.

TABLE III. COMPARISON OF IQRF MESH SOLUTION WITH RELEVANT SELECTED TECHNOLOGIES (EUROPE).

\begin{tabular}{|c|c|c|c|}
\hline & IQRF & Wi-Fi MESH & HaLow \\
\hline Frequency [MHz] & $433 / 868$ & $2400 / 5000$ & sub-GHz \\
\hline Throughput [kbs] & $<20$ & 2000 & up to 7800 \\
\hline Sensitivity [dBm] & -104 & -82 & -92 \\
\hline Range [m] & up to 1000 & up to 50 & up to 1000 \\
\hline Latency [ms] & 400 & $>500$ & 100 \\
\hline Consumption & up to $15 \mu \mathrm{A}$ & no LP & $>100 \mathrm{~mA}$ \\
\hline Security & AES-128 & SEA+PMK & WPA2/3 \\
\hline Topology* & s, t, m & m & s, t \\
\hline Sources & \multicolumn{5}{|c|}{$[\mathbf{1 4}],[53],[54],[55],[56],[57]$} \\
\hline
\end{tabular}

*Note: $s, t, m$ are for the star, tree, mesh topology.

The survey with LPWAN technologies and IQRF is in [43]. Moreover, the LPWAN technologies are in category MAN/WAN and not LAN/MAN. There might be one exception in the private LoRaWAN. The comparison with this technology is displayed in Table IV.

TABLE IV. COMPARISON OF IQRF MESH SOLUTION WITH RELEVANT SELECTED TECHNOLOGIES (EUROPE).

\begin{tabular}{|c|c|c|}
\hline & IQRF & LoRaWAN \\
\hline Frequency [MHz] & $433 / 868$ & 868 \\
\hline Throughput [kbs] & $<20$ & $<50$ \\
\hline Sensitivity [dBm] & -104 & -123 \\
\hline Range [m] & up to 1000 & $<20000$ \\
\hline Latency [ms] & 400 & $>1000$ \\
\hline Consumption & up to $15 \mu \mathrm{A}$ & $<1 \mathrm{~mA}$ \\
\hline Security & AES-128 & AES-128 \\
\hline Topology* & s, t, m [14], [43] \\
\hline \multicolumn{2}{|c|}{ Sources } & s \\
\hline
\end{tabular}

*Note: $s, t, m$ are for the star, tree, mesh topology.
The higher sensitivity from WAN application gives to LoRaWAN significant advantages in signal propagation and communication range and the throughput is comparable. However, the advantages of IQRF lies for this case in power-consumption and latency.

The technological comparison shows the uniqueness of IQRF technology in all cases. Choosing the right technology is not a simple task, but we provide a clear establishment for the IQRF between other solutions and highlight the main differences. This should help in the future decision making.

\section{CONCLUSIONS}

We successfully tracked the history of IQRF development from its early time to its current version. Moreover, we brought the in-depth analysis for the state of the art and showed the most important research direction in the field. We reviewed most of the published scientific papers focused on IQRF technology and summarized their findings. However, the most important part of this article is our original simulations and experimental measurements. We selected three main scenarios: indoor, open-space outdoor and urban-area outdoor. These scenarios cover most of the new applications in the area of Smart Grid, Smart City, and Smart Home, together with IoT and M2M, in which IQRF has its own place. The biggest advantages of IQRF are network stability, simple and fast convergence (without loss-rate), sufficient security level (AES-128) and very low power consumption per node (up to $15 \mu \mathrm{A}$ ). The biggest disadvantage compared to other relevant technologies is the small communication range, which may be a big limitation for outdoor scenarios and smaller networks without a sufficient number of nodes for MESH topology. The most suitable scenarios for outdoor use of IQRF would be so called sensor nest (networks with high density of nodes).

\section{REFERENCES}

[1] A. Goldsmith, Wireless communications. Cambridge university press, 2005. DOI: 10.1017/CBO9780511841224.

[2] A. G. Bell, "The photophone", Journal of the Franklin Institute, vol. 110, no. 4, pp. 237-248, 1880. DOI: 10.1016/00160032(80)90543-8.

[3] G. Marconi, "Wireless telegraphy", Journal of the Institution of Electrical Engineers, vol. 28, no. 139, pp. 273-290, 1899. DOI: 10.1049/jiee-1.1899.0010.

[4] A. Bassi, M. Bauer, M. Fiedler, T. Kramp, R. Kranenburg, S. Lange, S. Meissner, Enabling things to talk. Springer, 2016. DOI: 10.1007/978-3-642-40403-0.

[5] C. Anton-Haro, M. Dohler. Machine-to-machine (M2M) communications: architecture, performance and applications. Elsevier, 2014.

[6] P. B. Andersen, E. B. Hauksson, A. B. Pedersen, D. Gantenbein, B. Jansen, C. A. Andersen, J. Dall, Smart Grid Applications, Communications, and Security. Wiley-VCH, 2012.

[7] M. Deakin, Smart cities: governing, modelling and analysing the transition. Routledge, 2013. DOI: 10.4324/9780203076224.

[8] R. Harper, Inside the smart home. Springer Science \& Business Media, Springer-Verlag London, 2006.

[9] A. Gilchrist, Industry 4.0: the industrial internet of things. 2016.

[10] V. Markevičius, D. Navikas, D. Andriukaitis, M. Čepènas, A. Valinevičius, M. Žilys, R. Malekian, A. Janeliauskas, W. Walendziuk, A. Idzkowski, "Two thermocouples low power wireless sensors network", AEU-International journal of electronics and communications, vol. 84, pp. 242-250, 2017. DOI: 10.1016/j.aeue.2017.11.032.

[11] Ning Ye, Zhong-qin Wang, Ru-chuan Wang, Ting-ting Zhao, R. Malekian, D. Andriukaitis, A. Valinevicius, D. Navikas, V. Markevicius, "A Hidden Markov Model Combined with RFID-Based 
Sensors for Accurate Vehicle Route Prediction International Journal of Ad hoc and Ubiquitous Computing, vol. 23, iss. 1-2, p. 124-133, 2016. DOI: 10.1504/IJAHUC.2016.078473.

[12] R. A. Santos, et al., "Hybrid routing algorithm for emergency and rural wireless networks", Elektronika ir Elektrotechnika, vol. 89 no. 1 , pp. 3-8, 2009.

[13] D. J. Cook, S. K. Das, Smart Environments: Technologies, Protocols and Applications, chpt., Wireless Sensor Networks, USA: John Wiley, 2004. DOI: 10.1002/047168659X.

[14] IQRF. Technology for wireless. [Online]. Available: https://www.iqrf.org/technology/

[15] R. Kuchta, R. Vrba, V. Sulc, "Smart platform for wireless communication-Case study", in Proc. 7th IEEE Int. Conf. Networking, 2008, pp. 117-120. DOI: 10.1109/ICN.2008.114.

[16] R. Kuchta, R. Vrba, V. Sulc, "New wireless communication platform for home automation-case study", in Proc. 3rd Int. Conf. Digital Society, 2009, pp. 343-346. DOI: 10.1109/ICDS.2009.44.

[17] R. Kuchta, R. Vrba, V. Sulc, "IQRF smart wireless platform for home automation: a case study", in Proc. 5th IEEE Int. Conf. Wireless and Mobile Communications, 2009, pp. 168-173. DOI: 10.1109/ICWMC.2009.35.

[18] P. Seflova, V. Sulc, R. Spinar, "IQRF wireless technology utilizing IQMESH protocol", in Proc. 35th IEEE Int. Conf. Telecommunications and Signal Processing, 2012, pp. 101-104. DOI: 10.1109/TSP.2012.6256261

[19] IQRF, “OS Operating System: User's Guide (v2.00)". 2008

[20] V. Sulc, R. Kuchta, R. Vrba, "IQMESH implementation in IQRF wireless communication platform", in Proc. IEEE 2nd Int. Conf. Advances in Mesh Networks, 2009, pp. 62-65. DOI: 10.1109/MESH.2009.18.

[21] T. W. Oktaviani, "Perancangan user interface berbasis web untuk home automation gateway berbasis IQRF TR53B", Jurnal Nasional Teknik Elektro dan Teknologi Informasi, vol. 3, no. 3, pp. 179-186, 2014. DOI: 10.22146/jnteti.v3i3.85.

[22] B. Nugroho, W. Widyawan, E. Firmansyah, "Pegembangan Home Automation Berbasis Jaringan Sensor Nirkabel IQRF TR-52B Untuk Lampu Penerangan", in Prosiding SNST Fakultas Teknik, vol. 1, no. 1, pp. 15-19, 2015. [Online]. Available: https://www.publikasiilmiah.unwahas.ac.id/index.php/PROSIDING_S NST_FT/article/view/1178/1284

[23] V. Sulc, R. Kuchta, R. Vrba, "IQRF smart house-a case study", in Proc. 3rd IEEE Int. Conf. Advances in Mesh Networks, 2010, pp. 103-108. DOI: 10.1109/MESH.2010.17.

[24] M. Pies, R. Hajovsky, M. Latocha, S. Ozana, "Radio telemetry unit for online monitoring system at mining dumps", Applied Mechanics and Materials, vol. 548-549, pp. 736-743, 2014. DOI: 10.4028/www.scientific.net/AMM.548-549.736.

[25] S. B. Wibowo, G. D. Putra, B. S. Hantono, "Development of embedded gateway for Wireless Sensor Network and Internet Protocol interoperability", in Proc. 6th IEEE Int. Conf. Information Technology and Electrical Engineering, 2014, pp. 1-4. DOI: 10.1109/ICITEED.2014.7007920.

[26] P. Bazydlo, S. Dabrowski, R. Szewczyk, "Distributed temperature and humidity measurement system utilizing IQMESH wireless routing algorithms", Advances in Intelligent Systems and Computing (Progress in Automation, Robotics and Measuring Techniques), vol. 352, pp. 1-9, 2015. DOI: 10.1007/978-3-319-15835-8_1.

[27] P. Bazydlo, S. Dabrowski, R. Szewczyk, "Wireless temperature measurement system based on the IQRF platform", Advances in Intelligent Systems and Computing (Mechatronics-Ideas for Industrial Application), vol. 317, pp. 281-288, 2015. DOI: 10.1007/978-3-31910990-9_25.

[28] R. Hajovsky, M. Pies, "Use of IQRF technology for large monitoring systems", IFAC-PapersOnLine, vol. 48, no. 4, pp. 486-491, 2015. DOI: 10.1016/j.ifacol.2015.07.082.

[29] M. Pies, R. Hajovsky, "Use of IQRF technology for detection of construction inclination", in Proc. 6th Int. Eurasian Conf. Mathematical Sciences and Applications, 2016, vol. 1738, pp. 1-4. DOI: $10.1063 / 1.4952357$.

[30] V. Hartova, J. Hart, "Modern methods of monitoring variables for racehorses", in Proc. $16^{\text {th }}$ Int. Scientific Conf. Engineering for Rural Development, 2017, pp. 1018-1023. DOI: 10.22616/ERDev2017.16.N216.

[31] R. Hajovsky, M. Pies, M. Stankus, "Options of monitoring the state of protection networks", in Proc. Int. Conf. Mobile and Wireless Technology, 2017, pp. 157-165. DOI: 10.1007/978-981-10-5281117.
[32] M. Pies, R. Hajovsky, "Monitoring environmental variables through intelligent lamps", in Proc. Int. Conf. Mobile and Wireless Technology, 2017, pp. 148-156. DOI: 10.1007/978-981-10-52811 1_16.

[33] M. Pies, R. Hajovsky, "Using the IQRF technology for the internet of things: case studies", in Proc. Int. Conf. Mobile and Wireless Technology, 2017, pp. 274-283. DOI: 10.1007/978-981-10-5281131 .

[34] D. Ursutiu, C. Samoila, V. Jinga, "Creative developments in LabVIEW student training: (Creativity laboratory - LabVIEW academy)", in Proc. 4th IEEE Int. Conf. Experiment@, 2017, pp. 309-312. DOI: 10.1109/EXPAT.2017.7984399.

[35] D. Ursutiu, et al. "The future of "hardware-software reconfigurable", in Proc. Int. Conf. Interactive Collaborative Learning, 2016, pp. 269 275. DOI: 10.1007/978-3-319-50340-0_23.

[36] IQRF, OS Operating System: User's Guide (v3.00). 2011. [Online]. Available: http://www.iqrf.org/weben/downloads.php?id=231

[37] IQRF, OS Operating System: User's Guide (v3.04D). 2013. [Online]. Available: http://www.iqrf.org/weben/downloads.php?id=338

[38] IQRF, OS Operating System: User's Guide (v3.05D). 2014. [Online] Available: http://www.iqrf.org/weben/downloads.php?id=375

[39] IQRF, OS Operating System: User's Guide (v3.07D). 2015. [Online]. Available: http://www.iqrf.org/weben/downloads.php?id=462

[40] IQRF, OS Operating System: User's Guide (v3.08D). 2016. [Online] Available: http://www.iqrf.org/weben/downloads.php?id=494

[41] IQRF, OS Operating System: User's Guide (v4.00D). 2017. [Online] Available: http://www.iqrf.org/weben/downloads.php?id=512

[42] AlarmNet, Network Overview, 2005. [Online]. Available: http://library.ademconet.com/MWT/fs2/7810IR/AlarmNet-networkoverview.PDF

[43] L. Krupka, L. Vojtech, M. Neruda, "The issue of LPWAN technology coexistence in IoT environment", in Proc. 17th IEEE Int. Conf. Mechatronics-Mechatronika, 2016, pp. 1-8. [Online]. Available: http://ieeexplore.ieee.org/abstract/document/7827866/

[44] S. M. Darroudi, C. Gomez, "Bluetooth low energy mesh networks: a survey", vol. 17, no. 7, p. 1467, 2017. DOI: 10.3390/s17071467.

[45] Y. Wang, et al., "Energy-aware restricted access window control with retransmission scheme for IEEE 802.11ah (Wi-Fi HaLow) based networks", in Proc. 13th IEEE Annual Conf. Wireless On-Demand Network Systems and Services, 2017, pp. 69-76. DOI: 10.1109/WONS.2017.7888774.

[46] Remcom. Wireless InSite, (C) 2018. [Online]. Available: https://www.remcom.com/wireless-insite-em-propagation-software/

[47] Link Technologies. Tover Coverage: Quickly Create RF Propagation Maps for Anywhere in the World!, (C) 2018. [Online]. Available: https://www.towercoverage.com/

[48] IQRF, “TR-72D: RF Transceiver Module”, datasheet, 2017. [Online]. Available: http://iqrf.org/weben/downloads.php?id=337

[49] IQRF, "GW-ETH-02A: IQRF Ethernet Gateway (firmware version 3.10)", user's guide, 2017. [Online]. Available: http://www.iqrf.org/ weben/downloads.php?id=381

[50] Digi, "Indoor Path Loss", application note (XST-AN005a-Indoor), 2012. [Online]. Available: http://ftp1.digi.com/support/images/XSTAN005a-IndoorPathLoss.pdf

[51] R. Rudd, K. Craig, M. Ganley, R. Hartless, "Building materials and propagation: final report", 2014. [Online]. Available: https://www.ofcom.org.uk/_data/assets/pdf_file/0016/84022/buildin g_materials_and_propagation.pdf

[52] Bluetooth. Bluetooth Low Energy Regulatory Aspects (Version 10r00). 2011.

[53] G. R. Hiertz, et al., "IEEE 802.11s: the WLAN mesh standard", IEEE Wireless Communications, vol. 17 no. 1, 2010. DOI: 10.1109/MWC.2010.5416357.

[54] S. Aust, R. V. Prasad, I. G. M. M. Niemegeers, "IEEE 802.11 ah: Advantages in standards and further challenges for sub $1 \mathrm{GHz}$ Wi-Fi", in Proc. 2012 IEEE Int. Conf. Communications, 2012, pp. 68856889. DOI: $10.1109 /$ ICC.2012.6364903.

[55] T. Adame, et al., "IEEE 802.11 ah: the WiFi approach for M2M communications", IEEE Wireless Communications, vol. 21 no. 6 , pp. 144-152, 2014. DOI: 10.1109/MWC.2014.7000982.

[56] G. R. Hiertz, et al., "IEEE 802.11s MAC fundamentals", in Proc. 2007 IEEE Int. Conf. Mobile Adhoc and Sensor Systems, 2007, pp. 18. DOI: 10.1109/MOBHOC.2007.4428715.

[57] S. Chakraborty, "Analyzing peer specific power saving in IEEE 802.11s through queuing Petri Nets: some insights and future research directions", IEEE Trans. Wireless Communications, vol. 15, no. 5, pp. 3746-3754, 2016. DOI: 10.1109/TWC.2016.2527694. 